\title{
Análise psicométrica da escala de heteroavaliação de estilos de liderança
}

Luciana Mourão. Universidade Salgado de Oliveira.

Cristiane Faiad. Universidade de Brasília.

Francisco Antonio Coelho Junior. Universidade de Brasília.

\section{Resumo}

Liderança é um tema complexo e bastante pesquisado em comportamento organizacional. Este artigo objetiva relatar o processo de criação e testes iniciais de validade de uma escala de estilos de liderança. Foram realizados três estudos, com a participação de 2.510 trabalhadores. O primeiro estudo, uma análise cruzada, aponta evidências de validade psicométrica. O segundo compreende a análise confirmatória da estrutura fatorial da escala. Por fim, o terceiro estudo apresenta a validade de critério a partir da análise da correlação de Pearson com a satisfação no trabalho. Os resultados iniciais indicaram uma escala de 15 itens e estrutura bidimensional, com os fatores Foco nas Pessoas e Foco nos Resultados. Uma primeira análise de equação estrutural revelou um ajuste marginal do modelo hipotetizado, com sugestão de supressão de quatro itens, tendo confirmado a estrutura fatorial com bom ajuste. A escala apresentou correlação positiva e significativa com a satisfação no trabalho.

Palavras-chave: análise fatorial confirmatória; estilos de liderança; medidas.

\begin{abstract}
Psychometric Analysis of the Leadership Styles Hetero-assessment Scale. Leadership is a complex issue and widely researched in organizational behavior. This article aims to describe the process of creation and initial tests of validity of a leadership styles scale. We conducted three studies, involving 2,510 workers. The first study, a cross-analysis, shows evidence of psychometric validity. The second study comprises the confirmatory factor analysis of the scale. Finally, the third one presents the criterion validity based on the analysis of Pearson correlation with job satisfaction. Initial results indicated a scale of 15 items and twodimensional structure with the factors Focus on People and Focus on Results. A first analysis of structural equation revealed a marginal adjustment of the hypothesized model, with suggested deletion of four items, and confirmed the factor structure tested with good fit. The scale showed a positive and significant correlation with job satisfaction.
\end{abstract}

Keywords: confirmatory factor analysis; leadership styles; measures.

\section{Resumen}

Análisis psicométrico de los estilos de liderazgo de Escala hetero. El liderazgo es un tema complexo y bastante investigado en comportamiento organizacional. Este artículo objetiva relatar el proceso de creación y las pruebas iniciales de la validación de una escala de estilos de liderazgo. Se realizaron tres estudios, en los cuales participaron 2.510 trabajadores. El primer estudio, que fue un análisis cruzado, señala las evidencias de la validación psicométrica. El segundo comprende el análisis confirmatorio de la estructura factorial de la escala. Por último, el tercer estudio presenta la validación de criterio a partir del análisis de la correlación con la satisfacción en el trabajo. Los resultados iniciales indicaron una escala de 15 ítems y una estructura bidimensional, con los factores Foco en las Personas y Foco en los Resultados. Un primer análisis de la ecuación estructural reveló un ajuste marginal del modelo de hipótesis, con sugestión de supresión de cuatro ítems, confirmándose la estructura factorial probada con buen ajuste. La escala presentó correlación positiva y significativa con la satisfacción en el trabajo.

Palabras clave: análisis factorial confirmatorio; estilos de liderazgo; medidas. 
O tema liderança tem sido estudado desde as civilizações antigas, mas o interesse no mesmo tem sido renovado e reconhecido como atual, com crescentes pesquisas na área (Day, \& Dragoni, 2015; Dionne et al., 2014; Parry, Mumford, Bower, \& Watts, 2014). Estudos recentes sobre a temática apontam que a liderança exerce efeito sobre o desempenho da organização, quer seja efeito direto (Cherian \& Farouq, 2013); quer seja moderado por outras variáveis (Wu, 2014). Além disso, os estilos de liderança também estão relacionados ao comprometimento dos empregados (Garg \& Ramjee, 2013; Zanini, Santos, \& Lima, 2015); à satisfação no trabalho (Chavaglia, Dela Coleta, Dela Coleta, Mendes, \& Trevizan, 2013; Judge \& Kammeyer-Mueller, 2012), ao empowerment, contexto e desempenho de equipes de trabalho (Dias \& Borges, 2015; Tuuli, Rowlinson, Fellows, \& Liu, 2012); bem como à aprendizagem organizacional, sobretudo em termos de capacidade de absorção (Sun \& Anderson, 2012); e aprendizagem no trabalho, voltada para as estratégias de aprendizagem (Lins \& Borges-Andrade, 2014) ou para os resultados de treinamento (Silva \& Mourão, 2015).

Em relação aos estudos empíricos, Fonseca, Porto e Borges-Andrade (2015) realizaram uma revisão sistemática da literatura nacional a partir de 35 relatos de pesquisas sobre papéis e perfis de liderança, contemplando a busca em 15 periódicos nacionais de Psicologia e Administração no período de 1996 a 2013. Os resultados apontam para um predomínio de estudos transversais, exploratórios, com métodos qualitativos, voltados para o setor privado da economia e para o segmento de serviços. A produção científica brasileira sobre liderança está defasada quando comparada a outras áreas do campo do comportamento organizacional e quando comparada às pesquisas feitas no exterior (Fonseca et al., 2015).

Uma revisão das principais teorias acerca do fenômeno da liderança é apresentada por Rodrigues, Ferreira e Mourão (2014), que percorrem desde a teoria com base nos traços até as teorias processuais, que são as predominantes nas pesquisas atuais. As autoras sinalizam o papel de destaque da figura dos líderes na construção de espaços nas organizações e na eficácia dos grupos de trabalho, a despeito da diversidade teórica sobre liderança. Tal diversidade conduz a uma variada nomenclatura, por exemplo, liderança carismática versus burocrática (Gottlieb, 2012), liderança transacional e transformacional (Garg \& Ramjee, 2013; Sun \& Anderson, 2012), liderança autocrática, democrática/participativa, visionário-inspiracional e laissez-faire (Hunt, 2010; Lok \& Crawford, 2004).

Do ponto de vista conceitual, diferentes definições têm sido apresentadas para a liderança, embora o conceito ainda seja considerado vago (Spicker, 2012). Apesar das divergências, a questão do processo de influência e envolvimento de pessoas costuma ser frequente nas diferentes definições. Assim, neste estudo elegeu-se como parâmetro a definição de Yukl (1989, p. 253), "liderança é um construto complexo e multifacetado, que influencia tarefas e estratégias, bem como o compromisso e o cumprimento de tarefas para atingir os objetivos. Influencia ainda a manutenção e identificação de equipes, bem como a cultura da organização".

O presente artigo apresenta uma escala de estilos gerenciais baseada na teoria de Blake e Mouton (1964), que pertence à corrente teórica de liderança contingencial - também denominada abordagem situacional. A ideia central dessa corrente é de que o contexto influencia no estilo de liderança e que, portanto, pessoas podem ter diferentes estilos de liderança a depender da situação ou do contexto.

Nessa linha, estudos de pesquisadores da Ohio University buscaram articular o desempenho de líderes com a eficácia empresarial e apontaram que parte das atividades do líder encontrava-se ligada a tarefas e outra parte a pessoas, configurandose duas predisposições ou dimensões distintas da liderança (Sant'anna, Campos, \& Lótfi, 2012). Estudos de Likert (1961), na Universidade de Michigan, chegaram a resultados semelhantes, propondo estilos de liderança denominados "orientação para o empregado" e "orientação para a produção".

O Grid Gerencial de Blake e Mouton (1964) fundamenta-se, portanto, nesses estudos anteriores que definiam duas dimensões básicas da liderança: orientação para a produção e orientação para as pessoas. De acordo com o Grid desenvolvido pelos autores, cada uma dessas dimensões pode variar numa escala de 1 a 9, em uma matriz onde a orientação para essas duas dimensões se cruza, sendo apontados os estilos de acordo com a posição que o líder ocuparia em termos de preocupação com a produção/resultados e preocupação com as pessoas. Assim, um líder, 9,1 teria preocupação máxima com a produção (9) e preocupação mínima com as pessoas (1). Por outro lado, um líder 2,8 
teria preocupação baixa com a produção (2) e elevada com as pessoas (8). Dentre as 81 posições possíveis do Grid Gerencial, Blake e Mouton (1964), destacam cinco, correspondentes à posição central e às quatro extremidades da matriz, ou seja, estilo 9,1 (Gerência com autoridade/obediência); estilo 1,9 (Gerência de clube campestre); estilo 1,1 (Gerência empobrecida); estilo 9,9 (Gerência de equipe); e estilo 5,5 (Gerência do homem organizacional). Essa operacionalização da teoria de Blake e Mouton (1964) sob essas cinco perspectivas ou estilos buscou mostrar as possibilidades, a partir de um modelo matricial, de definição e desenvolvimento de um conjunto de estilos de liderança. A fim de facilitar a compreensão dos mesmos, os autores atribuíram alguns nomes para essas cinco composições principais, a saber: liderança empobrecida (baixa produção e baixa preocupação com as pessoas), liderança "clube de campo" (alta preocupação com as pessoas/baixa produção), liderança de tarefa (alta produção e baixa preocupação com as pessoas), liderança meio termo (produção mediana e média preocupação com as pessoas) e liderança em equipe (alta produção e alta preocupação com as pessoas).

Essa identificação da posição do gestor no Grid Gerencial - com seus respectivos nomes - serviria, na proposta de Blake e Mouton (1964), não só para se conhecer o estilo atual de determinado líder, mas também para identificar as suas necessidades de treinamento, a fim de melhorar as habilidades de liderança, quer seja nas deficiências quanto à falta de orientação para a produção, quer seja na falta de orientação para as pessoas (Silva Junior, Oliveira, \& Vasconcelos, 2010). Assim é que essa teoria propõe uma combinação ideal a ser buscada no desenvolvimento de líderes - a qual caracteriza-se pela Gerência de equipe (alta orientação para a produção e alta orientação para as pessoas).

A perspectiva de Blake e Mouton (1964) apresenta, portanto, convergência com a proposta de Bennis (1989), em termos da perspectiva de transformação do líder, a partir de processos de mudanças baseados em aprendizagem e conhecimento (Silva Junior et al., 2010). Nesse sentido, apesar de as teorias de liderança atuais estarem bastante voltadas para os processos (Garg \& Ramjee, 2013), a teoria de Blake e Mouton não estaria superada, uma vez que, de forma orgânica, os estilos de liderança emergem num dado contexto, que também engloba processos e trocas entre líderes e liderados, sendo prevalentes as abordagens mais integrativas das teorias de liderança (Avolio, 2007; Larsson \& Eid, 2012).
Além disso, observa-se, especialmente na construção de medidas disponibilizadas na literatura, que as dimensões usualmente associadas à liderança remontam à perspectiva clássica de Blake e Mouton (1964), no tocante à relação do líder com a equipe (líder-equipe), à realização das rotinas de trabalho (lídertarefa) e ao desenvolvimento das relações interpessoais (líder-pessoa).

Em virtude disto, adotou-se como parâmetro a proposição de uma nova medida brasileira à luz desta teoria clássica, ainda tida como referência neste tema. Couto (2015) aponta que ainda são poucos os instrumentos brasileiros validados psicometricamente sobre liderança (por exemplo, Melo 2004), e que estes dão ênfase a aspectos contingenciais da liderança com relação ao perfil esperado de um líder (se transformacional, se carismático, se visionário, se liderança coaching, e outros tipos) e, também, consideram a perspectiva de maturidade psicológica dos liderados com foco na teoria de Hersey-Blanchard (1982).

Instrumentos específicos da teoria de Blake e Mouton (1964) não foram encontrados, a apesar de as dimensões contempladas nesta teoria permitirem um extenso número de combinações de estilos gerenciais, além de estarem presentes em teorias contemporâneas que também focalizam aspectos da tarefa e das relações interpessoais (Avolio, Walumbwa, \& Weber, 2009). Em virtude disto, propomos uma nova medida científica com base na teoria de Blake e Mouton, quer seja porque as dimensões de orientação para pessoas e para a resultados/produção continuam presentes no cenário laboral, quer seja porque oferecem uma possibilidade de diagnóstico da atuação da liderança que pode contribuir para o processo de desenvolvimento das mesmas, o que é amplamente buscado pelas organizações de trabalho.

Em relação aos instrumentos de medida de liderança, predominam as escalas baseadas na liderança transformacional, sobretudo com o uso do Multifactor Leadership Questionnarie - MLQ (Bass, 1985). Embora haja outras medidas como a de Scott (2010), que tem oito fatores (Suporte, Carisma, Inteligência, Liderança responsável, Visão, Integridade, Assunção de riscos e Desafios à tradição) e a de Gnambs e Batinic (2011), que apresentam evidências de validade convergente e discriminante para uma escala de estilos de liderança a partir das opiniões dos líderes (assertivas, polimórficas, monomórficas e envolventes). Na literatura nacional, há também artigos voltados para a testagem da validade 
psicométrica de escalas de liderança (Benedetti, Hanashiro, \& Popadiuk, 2004; Hernandez \& Voser, 2012; Melo, 2004; Souza, Lopez, Bornia, \& Alves, 2013). Contudo, nenhuma dessas medidas toma por base a teoria de Blake e Mouton (1964).

Além da ausência de instrumentos que permitam a análise matricial proposta pelos autores, a literatura de liderança contradiz a tendência, no campo da Psicologia, de escalas curtas (Romero, Villar, Gómez-Fraguela, \& López-Romero, 2012; Schweizer, 2011), que possam ser aplicadas em conjunto com outros instrumentos para relacionar os estilos de liderança a outras variáveis de comportamento organizacional ou de gestão de pessoas. Isso é importante porque poucos estudos de liderança estão relacionados à "realidade da vida cotidiana" (Bird \& Wang, 2013; Tupinambá, 2012) e também porque há um predomínio nas pesquisas nacionais de estudos qualitativos, com poucos estudos que apresentam os antecedentes, consequentes, mediadores e moderadores da liderança (Fonseca et al., 2015).

Outro aspecto apontado por Hunt (2010) e que merece ser trazido em um artigo de medida de estilos de liderança diz respeito à inadequação de escalas que consideram os estilos de liderança como construto com uma única dimensão e que medem o foco central do líder com variação em um continuum de dois polos: autocrático (foco nos interesses do patrão) e democrático (foco nos interesses dos empregados). O autor argumenta que a medida com uma dimensão única é insuficiente para explicar os estilos de liderança e defende o modelo proposto por Blake e Mouton (1964), que é bidimensional, considerando dois contínuos, um relativo à orientação para a produção/resultados e outro relativo à orientação para as pessoas/empregados.

Assim é que, embora a agenda de pesquisa sobre liderança apresentada por Fonseca et al. (2015) sugira que seja evitada uma proliferação nos instrumentos nacionais sobre liderança para obstar um excesso de medidas (como ocorre na literatura estrangeira), a escala desenvolvida e apresentada no presente artigo tem dois diferenciais importantes e que atendem a lacunas identificadas na agenda proposta por tais autores. 0 primeiro ponto diz respeito ao modelo matricial da teoria, pois apesar de ter apenas duas dimensões, estas podem ser combinadas entre si de forma a gerar vários estilos gerenciais (com destaque para cinco), o que permite ampliar as pesquisas quantitativas e a rede nomológica do construto. O segundo ponto fundamenta-se no fato de ser uma medida baseada em heteroavaliação, com o foco na percepção dos subordinados, o que permite reduzir possíveis vieses de desejabilidade social comuns a pesquisas de autoavaliação.

Assim, foram construídos itens, sob a perspectiva de avaliação dos subordinados, tendo como suporte teórico a classificação bidimensional proposta por Blake e Mouton (1964). A escolha dessa teoria de liderança para embasar a escala, dentre tantas outras disponíveis, se deu por três razões principais: (i) pela utilidade da mesma na compreensão dos estilos de liderança predominantes nas organizações, uma vez que aborda dois aspectos centrais nos processos de gestão - o foco nos resultados e o foco nas pessoas; (ii) pelo fato de ter apenas duas dimensões, o que caracteriza um instrumento simples e mais fácil tanto de ser aplicado, quanto de ser relacionado a outros construtos; e (iii) pelo fato de a combinação das duas dimensões garantir ao instrumento ampla gama de possibilidades de estilos de liderança, uma vez que os resultados podem ser interpretados à luz do Grid Gerencial.

A escala foi construída de forma que pudesse atender há alguns resultados de pesquisa que mostram que certos estilos de liderança são mais suscetíveis de serem eficazes em determinados regimes de emprego (Liu, Lepak, Takeuchi, \& Sims, 2003) e que eles dependem dos contextos em que as organizações se inserem (Manning, 2013, Tupinambá, 2012). Assim, a construção dos itens foi feita considerando diferentes regimes de emprego e contextos de trabalho.

Como foi construída a partir do modelo de estilos de liderança de Blake e Mouton (1964), a escala considera duas dimensões que podem ser assim descritas: (i) Liderança com foco nas pessoas - gestores que em suas ações demonstram preocupação com os relacionamentos interpessoais e com aspectos pessoais dos membros de sua equipe de trabalho, bem como com o desenvolvimento e a autonomia da equipe e de seus membros; (ii) Liderança com foco na produção/resultados - gestores que em suas ações demonstram preocupação com os resultados do trabalho, o cumprimento de prazos e metas, bem como com o feedback dos desempenhos individuais.

Para testar a validade de critério da medida, foi investigada a relação entre o estilo de liderança e a satisfação no trabalho. A relação entre satisfação no trabalho e estilo de liderança é amplamente prevista pela literatura da área. A Teoria Caminho-Objetivo especifica comportamentos de líderes que impulsionam o desempenho e a satisfação dos seguidores no trabalho 
(Araújo et al., 2013; House \& Delbecq, 1997) e a satisfação juntamente com compromisso formam uma dimensão da Escala de Liderança Carismática Socializada (Chavaglia et al., 2013). Vale ainda destacar que os estudos de Judge e Kammeyer-Mueller (2012) sobre atitudes no trabalho mostram que os comportamentos e estilos de liderança têm um poderoso efeito sobre a satisfação dos subordinados (correlação meta-analítica de 0,78). Assim, a relação entre estilo de liderança e satisfação no trabalho seria um bom indicador de validade de critério da escala aqui apresentada.

\section{Método}

Com o objetivo de se verificar a estrutura fatorial do instrumento, bem como sua replicação e estabilidade, foi aplicada, em um primeiro momento, a técnica de validação cruzada por meio de duas análises fatoriais exploratórias com base nas duas primeiras amostras. Em sequência, os dados de uma terceira amostra foram submetidos à modelagem de equações estruturais para confirmação da estrutura do instrumento. A validade de critério foi testada nesta mesma amostra, a partir da análise da relação entre o estilo de liderança e a satisfação no trabalho.

\section{Participantes}

Participaram da presente pesquisa 2.510 profissionais de 18 estados brasileiros, compreendendo as cinco macrorregiões do Brasil (Norte - 25,5\%; Nordeste - 26,4\%; Centro-Oeste - 30,8\%; Sudeste - $12,9 \%$ e Sul - 4,4\%), separados aleatoriamente em três amostras. As amostras foram compostas por 856,854 e 800 participantes, respectivamente e serão descritas a seguir.

A primeira amostra contou com $53,7 \%$ de homens, com idade média de 32,53 anos $(D P=9,61)$, sendo $49,7 \%$ com ensino médio e $43,4 \%$ com graduação. Quanto ao tamanho da empresa, 38,3\% trabalhavam em organizações com até 50 funcionários, 33,6\% em organizações com mais de 500 funcionários. Quanto à ocupação de cargo gerencial, 74\% indicaram não exercer essa função. Trata-se de organização eminentemente privada $(71,4 \%)$, com três quartos da amostra $(75,1 \%)$ voltada para a área de serviços. Quanto ao tempo de trabalho, 39,7\% estavam entre a faixa de 0 a 3 anos de trabalho, 24,6\% de 3 a 6 anos e $18,9 \%$ acima de 10 anos de trabalho.

A segunda amostra contou com 50,3\% homens, com idade média de 32,49 anos $(D P=8,18)$, sendo
$50,2 \%$ com ensino médio e $44,5 \%$ com graduação. Quanto ao tamanho da empresa, $41,8 \%$ atuavam em organizações com até 50 funcionários e 27,1\% em organizações grandes, com mais de 500 funcionários. Quanto à ocupação de cargo gerencial, $75,4 \%$ não exerce essa função. As organizações de trabalho eram predominantemente privadas (74,2\%), e com atuação na área de serviços (72,3\%). Um expressivo percentual de pesquisados tinha até três anos de trabalho $(42,3 \%)$ enquanto um quantitativo menor $(19,8 \%)$ tinha 10 ou mais anos de trabalho.

A terceira amostra contou com $55 \%$ de homens, a idade média foi de 32,59 anos ( $D P=9,33)$, sendo $53,3 \%$ com ensino médio e $42,7 \%$ com graduação. Pouco mais de um quarto da amostra $(27,5 \%)$ trabalhava em organizações pequenas com até 50 funcionários e mais da metade (52,9\%) atuava em grandes organizações com mais de 500 funcionários. Predominaram as organizações privadas $(70,6 \%)$, com foco na área de serviços $(69,4 \%)$. Quanto ao tempo de trabalho, a média foi de 7,18 anos e desvio-padrão de 8,18 anos, sendo que 322 (40,3\%) tinham até três anos de trabalho e $183(22,9 \%)$ mais de 10 anos trabalhados, com poucos pesquisados $(16,2 \%)$ que exerciam algum nível de função gerencial.

\section{Instrumentos}

A Escala de Heteroavaliação de Estilos de Liderança (EHEL) é composta por 15 itens, avaliados por meio de uma escala de ancoragem ( 0 = discordo totalmente a 10 = concordo totalmente). No questionário que continha a escala, foram também incluídas oito questões para avaliar características sociodemográficas.

Os itens da escala foram construídos tendo como base teórica o Modelo de Blake e Mouton (1964) que subdivide os estilos de liderança em: Liderança com Foco em Pessoas e Liderança com Foco na Produção/ Resultados. Os itens foram originados de duas fontes: entrevistas e grupo focal com trabalhadores de ambos os sexos, de variadas idades e categorias profissionais e análise de itens de outras escalas de liderança e estilos de gerenciamento, especificamente a partir dos instrumentos de Melo (2004) e W. Silva, Laros e Mourão (2007).

Para completar o processo de desenvolvimento da escala, foi realizada uma análise de juízes, que contou com a participação de oito especialistas que avaliaram a pertinência dos itens para a mensuração do construto, bem como a adequação dos mesmos às duas dimensões previstas no modelo teórico. Esses juízes foram cinco 
homens e três mulheres, todos professores de programas de pós-graduação no Brasil, nas áreas de Psicologia ou Administração, com mais de 10 anos de formados. Foi estabelecido o critério de concordância de $80 \%$ dos juízes para a manutenção dos itens das escalas. Nenhum item foi descartado nesta etapa, mas a redação de três deles foi aprimorada a fim de tornar mais compreensível os aspectos que pretendia investigar.

A Escala de Satisfação no Trabalho é composta por cinco questões, ancoradas numa escala de concordância, tipo Likert, variando de zero (discordo totalmente) a 10 (concordo totalmente). Foi desenvolvida foi validada por A. P. C. Silva e Ferreira (2009), com estrutura unifatorial, com explicação de $65,9 \%$ da variância total do instrumento. A consistência interna deste fator, calculada por meio do coeficiente Alfa de Cronbach, é de 0,89.

\section{Procedimentos}

O instrumento de coleta de dados foi aplicado em todas as regiões brasileiras, com o auxílio de uma rede formada por alunos de um curso de administração a distância. A aplicação foi dividida em duas formas: distribuição e recolhimento dos questionários em papel (82\% da amostra) e envio do questionário para resposta por e-mail (18\%). As instruções de preenchimento do questionário permitiam que o instrumento fosse autoaplicável. $O$ tempo de preenchimento era livre e a taxa de retorno foi de $68 \%$.

Para maior fidedignidade dos dados, foi estabelecido um sistema de acompanhamento do processo de coleta de dados. A equipe de pesquisadores recebeu treinamento para a aplicação dos questionários e um material com procedimentos detalhados que deveriam ser seguidos durante a coleta de dados. A cada seis pesquisadores havia um líder que recebia e conferia o preenchimento dos questionários e apresentava o relato para a coordenadora geral da pesquisa. 0 período de coleta de dados foi de quatro semanas, com acompanhamento diário do andamento das atividades dos pesquisadores. Aos participantes foi esclarecido o objetivo da pesquisa, garantido sigilo das respostas individuais, informada a possibilidade de desistência de participação em qualquer fase do processo, bem como disponibilizado acesso aos resultados da pesquisa.

As análises estatísticas foram feitas com apoio de três softwares: Statistical Package for the Social Science - SPPS 21.0, Factor 8.0 e Analysis of Moment Structures - AMOS 21.0. Foram realizadas análises descritivas e exploratórias para investigar a exatidão da entrada dos dados, a presença de casos extremos, a distribuição dos casos omissos, a distribuição de frequência, de acordo com as orientações de Tabachnick e Fidell (2007). Em seguida, iniciaram-se os testes de validade estatística do instrumento.

A primeira análise foi feita a partir de um procedimento de validação cruzada com os dois primeiros bancos de dados aleatórios, por meio da análise fatorial exploratória. Para a realização de tal análise, inicialmente, foram investigados como pressupostos a fatorabilidade da medida mediante a inspeção da matriz de correlações, o cálculo do índice de adequação da amostra (KMO) e o teste de esfericidade de Bartlett. Tais procedimentos deveriam indicar correlação entre todos os itens da escala, KMO acima de 0,70 e teste de esferecidade de Bartlett significativo (Tabachnick \& Fidell, 2007).

A seguir foi feita uma análise fatorial confirmatória, realizada com o terceiro banco de dados. Nesta análise foi utilizado o método de estimação Maximuum Likelihood, como forma de definição da normalidade multivariada, considerando que a escala de respostas era de 11 pontos e que houve assunção de normalidade na distribuição das respostas aos itens. Após a especificação e estimação do modelo, a sua adequação foi avaliada por um conjunto de índices de ajustamento: Qui-quadrado $\left(x^{2}\right)$, que como critério não deve ser significativo, a razão do qui-quadrado pelos graus de liberdade, tendo em vista que o qui-quadrado é muito influenciado pelo tamanho da amostra. O critério é que esta razão seja inferior a 5,0 (Byrne, 1989), sendo desejável que ela seja menor que 2,0 (Tabachnick \& Fidell, 2007). E outros índices de ajuste do modelo, a saber: o TLI - Tucker-Lewis Index e o CFI - Comparative Fit Index, considerandose como parâmetro de um modelo adequado valores acima de 0,90 (Hu \& Bentler, 1999). Por fim, foram utilizados índices de parcimônia do modelo, a saber: RMSEA - Root Mean Square Error of Approximation, e SRMR - Standardized Root Mean Residual. Valores de SRMR variam numa escala de zero a um, considerandose modelos adequados quando os valores são inferiores a 0,05 (Byrne, 1989), entretanto valores até 0,08 são aceitáveis (Hu \& Bentler, 1999).

Para a avaliação da validade de critério foi realizada uma correlação de Pearson, adotando-se como parâmetro a existência de correlação significativa, de intensidade moderada (entre 0,30 e 0,49 ) ou alta (igual ou superior a 0,50 ), conforme os parâmetros estabelecidos por Miles e Shevlin (2001). Além da correlação foi realizada uma regressão linear padrão para 
a qual se esperava que os fatores de liderança fossem preditores da satisfação com o trabalho.

\section{Resultados}

Os dados serão apresentados em duas fases, sendo a primeira referente à validação cruzada, realizada mediante análise fatorial exploratória dos dois primeiros bancos de dados. A segunda análise trata-se de uma análise fatorial confirmatória, realizada com o terceiro banco de dados. As análises fatoriais exploratórias serão apresentadas concomitantemente para melhor comparação da consistência do modelo fatorial proposto.

\section{Primeiro Estudo: Análise Exploratória - Validação Cruzada}

A análise de validação cruzada é uma técnica que tem como objetivo avaliar a replicação de uma estrutura fatorial, quando aplicada a diferentes amostras. Inicialmente, foi investigada a fatorabilidade da matriz de dados. Os resultados foram satisfatórios uma vez que foi identificada presença de correlações significativas entre todos os itens da escala em ambas as amostras, o KMO foi de 0,94/0,95 e o teste de esfericidade de Bartlett significativo, $X^{2}=6.973,6 / 7.792,6 ; p<0,001$ ).

A seguir, para a extração dos fatores foram considerados dois métodos: análises paralelas de Horn (1965) e o método da média mínima parcial (Minimum Average Partial, MAP), proposto por Velicer (1976), uma vez que ambos são bastante confiáveis (Damásio, 2012). O método das análises paralelas (valor dos autovalores empíricos nas duas amostras de 1,62/1,43 maior que o randômico de 1,18 ) também apontou a existência de dois fatores. O MAP também indicou dois fatores, pois a partir deste ponto a média começava a subir, indicando que a variância específica dos itens passaria a ser considerada.

Uma vez definido o número de fatores, testouse um possível impacto dos outliers multivariados na estrutura dos componentes e observou-se que não houve modificação na estrutura dos mesmos. Foi então realizada a extração de dois fatores, pelo método Principal axis Factoring (PAF), com rotação oblíqua Direct Oblimim, e tratamento de casos omissos pelo método pairwise.

Os dados da Tabela 1 indicam que as cargas fatoriais variaram de 0,33 a 0,92 e se mostraram próximas nas duas amostras, com uma mesma distribuição fatorial em ambas. Como nenhuma carga fatorial ficou abaixo de 0,32 (Tabachinick \& Fidell, 2007), não houve supressão de
Tabela 1. Resultados da PAF para as Duas Amostras.

\begin{tabular}{|c|c|c|c|c|}
\hline \multirow{3}{*}{ Itens } & \multicolumn{4}{|c|}{ Amostras } \\
\hline & 1 & 2 & 1 & 2 \\
\hline & \multicolumn{2}{|c|}{ Fator 1} & \multicolumn{2}{|c|}{ Fator 2} \\
\hline $\begin{array}{l}10 \text { - Busca atender as necessidades } \\
\text { pessoais de cada membro do } \\
\text { grupo. }\end{array}$ & 0,86 & 0,85 & & \\
\hline $\begin{array}{l}15 \text { - Mostra-se atento às demandas } \\
\text { dos empregados. }\end{array}$ & 0,82 & 0,92 & & \\
\hline $\begin{array}{l}1 \text { - Preocupa-se muito com o bem- } \\
\text { estar dos seus subordinados. }\end{array}$ & 0,74 & 0,75 & & \\
\hline $\begin{array}{l}3 \text { - Trata os funcionários de forma } \\
\text { amigável. }\end{array}$ & 0,64 & 0,53 & & \\
\hline $\begin{array}{l}5 \text { - Incentiva o desenvolvimento } \\
\text { pessoal da sua equipe. }\end{array}$ & 0,48 & 0,51 & & \\
\hline $\begin{array}{l}8 \text { - Deixa que cada um decida como } \\
\text { e quando fazer o seu trabalho. }\end{array}$ & 0,57 & 0,63 & & \\
\hline $\begin{array}{l}7 \text { - Tem facilidades no } \\
\text { relacionamento interpessoal. }\end{array}$ & 0,53 & 0,55 & & \\
\hline $\begin{array}{l}9 \text { - Enfatiza a importância do } \\
\text { desempenho do grupo. }\end{array}$ & 0,47 & 0,54 & & \\
\hline $\begin{array}{l}13 \text { - Enfatiza a importância do } \\
\text { desempenho de cada funcionário. }\end{array}$ & 0,37 & 0,46 & & \\
\hline $\begin{array}{l}14 \text { - Faz reuniões de trabalho para } \\
\text { falar sobre a equipe. }\end{array}$ & 0,43 & 0,47 & & \\
\hline $\begin{array}{l}11 \text { - Tem o foco bastante voltado } \\
\text { para os resultados do trabalho. }\end{array}$ & & & 0,84 & 0,78 \\
\hline $\begin{array}{l}4 \text { - Preocupa-se bastante com } \\
\text { o cumprimento das tarefas de } \\
\text { trabalho. }\end{array}$ & & & 0,80 & 0,78 \\
\hline $\begin{array}{l}12 \text { - É atento ao cumprimento dos } \\
\text { prazos do trabalho. }\end{array}$ & & & 0,69 & 0,73 \\
\hline $\begin{array}{l}2 \text { - Estabelece metas claras para os } \\
\text { funcionários cumprirem. }\end{array}$ & & & 0,58 & 0,52 \\
\hline $\begin{array}{l}\text { 6- Acompanha os resultados do } \\
\text { trabalho de cada funcionário. }\end{array}$ & & & 0,53 & 0,45 \\
\hline Autovalor & 7,25 & 7,80 & 1,62 & 1,43 \\
\hline \% Variância explicada & 48,33 & 52,00 & 10,80 & 9,53 \\
\hline № de itens & 10 & 10 & 05 & 05 \\
\hline Alpha & 0,90 & 0,91 & 0,84 & 0,85 \\
\hline Lambda & 0,90 & 0,91 & 0,83 & 0,85 \\
\hline
\end{tabular}

nenhum item. Os dados dos alfas de consistência interna também foram próximos, variando entre 0,83 e 0,90 e os autovalores foram quase os mesmos, sendo este um indicador de semelhança na estrutura fatorial para as duas amostras. A partir desta análise de consistência, foi realizada uma análise de interpretação teórica dos itens. O primeiro fator foi intitulado como Foco nas Pessoas, caracterizando líderes que tem como foco pessoas e equipes, em suas ações e intervenções no trabalho, já o segundo fator foi nomeado como Foco no Resultado, 
caracterizando líderes que se preocupam mais com o desempenho no trabalho e o cumprimento das tarefas por seus subordinados. Sendo assim, a estrutura fatorial proposta para a Escala de Heteroavaliação de Estilos de Liderança (EHEL), a partir da análise exploratória, foi de um instrumento de dois fatores, composto por 15 itens. Partiu-se, então, para testar essa estrutura por meio da análise fatorial confirmatória.

\section{Segundo Estudo: Análise Confirmatória}

Para a análise confirmatória do modelo proposto, com base na terceira amostra, foram testados alguns pressupostos. $\mathrm{Na}$ análise de dados omissos, observouse que nenhuma variável superou o valor de $2 \%$, sendo que os casos existentes foram substituídos pela média. Foram verificados os casos extremos e os pressupostos de linearidade, homocedasticidade, independência dos resíduos e normalidade. Os outliers (casos extremos) univariados foram analisados com base no parâmetro do escore $Z>3,29$, onde foram encontrados seis casos univariados, permanecendo 853 casos. Os outliers multivariados foram avaliados com base na distância de Mahalanobis, na identificação da tabela do $\chi^{2}$ com 40 graus de liberdade $(p<0,001)$, de valor crítico igual a 73,40. Foram identificados dois casos multivariados (Hair, Black, Babin, Anderson, \& Tatham, 2009), mas não houve efeito com a retirada dos mesmos para o $\mathrm{MEE}$, mantendo-se os casos no banco. Verificou-se assimetria em dois itens, contudo como essa assimetria era apenas moderada, manteve-se a métrica original dessas variáveis.

Uma primeira análise de equação estrutural revelou um ajuste marginal do modelo hipotetizado: $\chi^{2}$ $=962,75, \chi^{2} / \mathrm{DF}=10,81 ; \mathrm{CFI}=0,89, \mathrm{TLI}=0,87, \mathrm{RMSEA}=$ 0,11 . Houve sugestão de três ajustes ao modelo proposto, entre os erros dos itens 2 e 4 e dos itens dos itens 7 e 10 e dos itens 14 e 15 . Os valores da correlação dos erros entre estes pares de itens foram respectivamente de 0,39, 0,52 e 0,32. Os valores do Modification Indices - MI (valor que seria reduzido no qui-quadrado com o ingresso da correlação de erros entre os pares de itens) que sinalizaram para a correção dos erros tiveram as seguintes magnitudes: entre os erros dos itens 2 e 4 (MI = 57.387); entre os itens 7 e $10(\mathrm{Ml}=62.074)$ e entre os itens 14 e 15 ( $M I=18.242)$. O item 5, por sua vez, foi retirado em função de a carga fatorial do mesmo ter sido de 0,37 , portanto bem abaixo do cutoff mínimo de 0,50 exigido para análises fatoriais confirmatórias (Tabachinick \& Fidell, 2007).
Após esses ajustes os dados indicaram um expressivo decréscimo no $\chi^{2}=453,05$; e na razão crítica $\left(\chi^{2} / d f=6,20\right)$. Os demais indicadores de ajuste do modelo atingiram os seguintes valores: $\mathrm{CFI}=0,95 ; \mathrm{TLI}=0,94$; RMSEA $=0,08$ (intervalo de confiança de $95 \%=0,07$ a 0,09), confirmando a estrutura fatorial testada com bom ajuste. As correlações entre os erros de alguns itens do modelo inicial encontram explicação teórica e serão justificadas na seção de discussão. Nesse sentido, para uma escala mais parcimoniosa, optouse pela testagem da medida com a retirada de um item de cada uma das duas duplas, sendo excluído aquele cuja carga fatorial foi mais baixa.

A retirada dos três itens de menor carga fatorial de cada um dos pares de itens que tinham correlação entre os erros (especificamente os itens 2, 5 e 10) resultou em uma escala de 11 itens (sendo 7 relativos ao Fator Foco nas Pessoas e 4 relativos ao Fator Foco nos Resultados). A análise fatorial confirmatória revelou um modelo de bom ajuste para esta versão final da medida, a saber: $\chi^{2}=332,76, \chi^{2} / d f=7,7 ; \mathrm{CFI}=0,95, \mathrm{TLI}=0,93$, RMSEA $=$ $0,089(0,080-0,098)$.

Os resultados da estrutura testada (considerando a versão final com 11 itens) mostraram cargas fatoriais elevadas nos fatores, variando de 0,69 a 0,82 para o Fator Foco nas Pessoas e de 0,62 a 0,77 para o Fator Foco nos Resultados. Essas cargas fatoriais elevadas são um bom indicador de estabilidade do instrumento em aplicações futuras. Quanto ao grau de fidedignidade, adotou-se o Alpha de Cronbach e o Lambda 2 de Guttman, com resultados bastante satisfatórios tanto para o Fator Foco nas Pessoas (Alpha $=0,90$; Lambda $=0,90$ ) quanto para o Fator Foco nos Resultados (Alpha =0,82; Lambda =0,82).

Para testar a invariância da medida, foi realizada uma Análise Fatorial Confirmatória Multigrupo. A avaliação testou o pressuposto de invariância configural (configural invariance), também chamado de modelo irrestrito (unconstrained model) ou igualdade de forma (Damasio, 2013). Este modelo avalia em que medida a estrutura de um determinado instrumento é plausível para todos os grupos que estão sendo analisados. O teste indicou que a estrutura fatorial da Escala de Heteroavaliação de Estilos de Liderança mantém-se a mesma quando o instrumento é aplicado no formato de lápis e papel ou em survey eletrônico. Os itens que compuseram cada um dos fatores mantiveram-se inalterados (Fator Foco nas Pessoas - itens 1, 3, 7, 8, 9, 13 e 15; e Fator Foco nos Resultados - itens 4, 6, 11 e 12). Uma vez confirmada a estrutura da medida, o próximo passo foi testar a sua validade de critério. 


\section{Terceiro Estudo: Teste de validade de critério}

Finalmente, foi realizada uma correlação de Pearson e uma regressão para testar a relação entre os estilos de liderança e a satisfação no trabalho, a fim de estudar a validade de critério da medida. Os resultados da correlação mostraram que a satisfação no trabalho tem correlação de intensidade moderada com o estilo de lideranças com foco em resultados $(r=0,41, p<$ $0,001)$ e correlação de intensidade elevada com o estilo de lideranças com foco em pessoas $(r=0,62, p<0,001)$. $O$ resultado da regressão linear padrão aponta para um poder de predição exclusivamente do fator de Foco nas Pessoas ( $\beta=0,67 ; p<0,001)$, pois o fator Foco nos Resultados não apresentou resultados significativos $(\beta=$ $0,07 ; p<0,101)$. Os estilos de liderança foram capazes de explicar $38,4 \%$ da variância da satisfação no trabalho ( $F$ $=245,5 ; p<0,001)$. Dessa forma, confirma-se a validade de critério da medida de estilos de liderança apresentada no presente estudo.

\section{Discussão}

Os resultados desta pesquisa mostram que os testes iniciais de validade da escala de estilos de liderança baseada na teoria de Blake e Mouton (1964) apontam as evidências de validade psicométrica. Tal validade foi apontada nos procedimentos de validação cruzada da análise fatorial exploratória, nos resultados da análise confirmatória da estrutura fatorial, na invariância da medida se consideradas diferentes formas de aplicação (lápis e papel ou survey eletrônico) ou ainda na validade de critério.

As modificações ocorridas em função das indicações resultantes da análise fatorial confirmatória merecem discussão. As alterações feitas no modelo inicial são justificadas uma vez que há semelhança nos itens cujos erros estão correlacionados (item 2 - "Estabelece metas claras para os funcionários cumprirem" com item 4 - "Preocupa-se bastante com os resultados do trabalho"; item 7 "Tem facilidades no relacionamento interpessoal" com item 10 "Busca atender as necessidades pessoais de cada membro do grupo", bem como na dupla item 14 - "Faz reuniões de trabalho para falar sobre a equipe" e item 15 - "Mostrase atento às demandas dos empregados").

A inclusão desses itens semelhantes na versão inicial da escala se deu em função das entrevistas realizadas para a construção do instrumento, em que trabalhadores de diferentes contextos laborais apresentavam depoimentos que tinham conteúdo semelhante, mas apresentavam uma forma de apresentação distinta. Por exemplo, o item 2 "Estabelece metas claras para os funcionários cumprirem" foi proveniente de depoimentos de trabalhadores ligados à iniciativa privada, enquanto o item 4 - "Preocupa-se bastante com os resultados do trabalho", foi comum a trabalhadores de diferentes contextos. Da mesma forma, o item 14 "Faz reuniões de trabalho para falar sobre a equipe" foi proveniente do discurso de trabalhadores de nível superior e, sobretudo, do setor terciário da economia. Já o item 15 - "Mostra-se atento às demandas dos empregados" foi mais comum ao conjunto dos depoimentos, além de seu conteúdo estar presente em outras escalas de liderança.

Como a proposta do instrumento era atender a trabalhadores de diferentes níveis de escolaridade e também situados em distintos contextos laborais, a opção inicial na etapa de construção da medida foi a de deixar itens, ainda que semelhantes, mas que compreendessem o que emergiu dos variados perfis de trabalhadores e contextos de trabalho. O resultado das análises estatísticas, contudo, mostraram que uma versão mais parcimoniosa da medida seria mais indicada, uma vez que as correlações entre os erros de tais itens indicam a semelhança de interpretação que os participantes tiveram acerca de seus conteúdos. Assim, a versão com 11 itens preserva a essência do conteúdo que se quer mensurar, ao tempo em que sinaliza que os itens com maior carga fatorial são justamente aqueles que aparecem de forma mais generalizada no corpus das entrevistas, sendo estes suficientes para a mensuração de cada uma das dimensões propostas pela escala.

A validade de critério também foi atestada a partir da correlação positiva e significativa de ambas as dimensões com a satisfação no trabalho, o que confirma os resultados apontados por Lok e Crawford (2004). O fato de a dimensão de liderança com foco nas pessoas ser preditora da satisfação no trabalho confirma achados anteriores da literatura que apontam a relação entre satisfação no trabalho e estilo de liderança (Araújo et al., 2013; Chavaglia et al., 2013; House \& Delbecq, 1997; Judge \& Kammeyer-Mueller, 2012).

Assim, a escala de Estilos de Liderança resultou em uma medida de 11 itens e estrutura bidimensional, com os fatores Foco nas Pessoas (sete itens) e Foco nos Resultados (quatro itens), com bons ajustes do modelo que confirmam a sua estrutura fatorial. A medida atende à crítica feita por Hunt (2010) de que medidas de estilos 
de liderança como construto com uma única dimensão são insuficientes para explicar os estilos de liderança. Nesse sentido, a defesa que Hunt (2010) faz do modelo proposto por Blake e Mouton (1964), pode ser apontada como uma das vantagens da escala, uma vez que ela possui natureza bidimensional e matricial.

A mensuração a partir dos contínuos de foco em resultados e foco em pessoas permite combinações das duas dimensões, gerando um extenso conjunto de possibilidades de estilos, sendo os mais característicos os que resultam dos quatro quadrantes, além daquele relativo à posição central, a saber: (i) líderes com alto foco em pessoas e também nos resultados; (ii) líderes com alto foco em pessoas e baixo foco em resultados; (iii) líderes com baixo foco em pessoas e alto foco em resultados; (iv) líderes com baixo foco em pessoas e também em resultados; e (v) líderes com foco mediano em pessoas e em resultados.

Deve-se também considerar que o fato de ser uma medida curta permite a sua aplicação em conjunto com outras escalas de comportamento organizacional ou de gestão de pessoas. Isso é especialmente importante porque os estilos de liderança podem ter um conjunto de implicações práticas para as equipes de trabalho e para as organizações (Bird \& Wang, 2013) e porque as pesquisas que investigam seus mediadores e moderadores ainda são escassas (Avolio et al., 2009; Fonseca et al., 2015).

Além disso, a medida pode ser aplicada em trabalhadores submetidos a diferentes regimes de emprego, pois seus itens foram construídos de forma a permitir respostas de trabalhadores provenientes de variados setores e situações de emprego. A simplicidade da escala permite que a mesma seja aplicada inclusive em trabalhadores de baixa escolaridade. Isso é importante porque, em diversos países, entre eles o Brasil, ainda há uma significativa massa de empregados que passaram poucos anos nas escolas.

Este estudo tem como limitação o fato de testar apenas alguns indicadores de validade da medida, sendo indicadas novas pesquisas que possam dar continuidade ao processo de busca de evidências de validade da escala. Sugere-se que em outros estudos sejam testados, por exemplo, a estabilidade temporal da medida, sua validade concorrente e discriminante.

\section{Referências}

Araújo, M. R. M., Silva, L. C. O., Gois, C. G., Sousa, D. A., Mendonça, P. F., Lima, T. C., \& Melo Neto, O. C. (2013). Preferência por tipos de liderança: um estudo em empresa sergipana do setor hoteleiro. Estudos de Psicologia, 18(2), 203-211. doi: 10.1590/S1413$-294 X 2013000200005$
Avolio, B. J. (2007). Promoting more integrative strategies for leadership theory-building. American Psychologist, 62(1), 25-33. doi: 10.1.1.467.7223\&rep=rep1\&type=pdf

Avolio, B. J., Walumbwa, F. O., \& Weber, T. J. (2009). Leadership: current theories, research, and future directions. Annual Review of Psychology, 60, 421-449. doi: 10.1146/annurev.psych.60.110707.163621

Bass, B. (1985). Leadership and performance beyond expectations. Nova lorque: The Free Press.

Benedetti, M. H., Hanashiro, D. M. M., \& Popadiuk, S. (2004). Liderança: uma relação com base no gerenciamento dos stakeholders, a partir da ótica dos liderados. Organizações \& Sociedade, 11(31), 59-76. doi: 10.1590/S1984-92302004000300004

Bennis, W. G. (1989). On becoming a leader. Nova lorque: Addison Wesley.

Bird, J. J., \& Wang, C. (2013). Superintendents describe their leadership styles: implications for practice. Management in Education, 27(1), 14-18. doi: 10.1177/0892020612459287.

Blake, R., \& Mouton, J. (1964). The managerial grid: the key to leadership excellence. Houston: Gulf Publishing Co.

Byrne, B. (1989). A primer of LISREL: basic applications programming for confirmatory factor analytic models. Nova lorque: Springer-Verlag.

Chavaglia, S. R. R., Dela Coleta, M. F., Dela Coleta, J. A., Mendes, I. A. C., \& Trevizan, M. A. (2013). Adaptação e validação da Escala de Liderança Carismática Socializada. Acta Paulista de Enfermagem, 26(5), 444-454. doi: 10.1590/S0103-21002013000500007.

Cherian, J., \& Farouq, S. (2013). Does effective leadership style drive financial performance of banks? Analysis in the context of UAE banking sector. International Journal of Economics and Finance, 5(7), 105-114. doi: 10.5539/ijef.v5n7p105.

Couto, F. J. (2015). Relações entre o estilo gerencial e a expressão de competências técnicas e comportamentais na Universidade de Brasilia (Dissertação de Mestrado). Universidade de Brasília, Brasília. Recuperado de http://repositorio.unb.br/handle/10482/19533

Damásio, B. F. (2012). Uso da análise fatorial exploratória em psicologia. Avaliação Psicológica, 11(2), 213-228.

Damásio, B. F. (2013). Contribuições da Análise Fatorial Confirmatória Multigrupo (AFCMG) na avaliação de invariância de instrumentos psicométricos. Psico-USF, 18(2), 211-220. doi: 10.1590/S141382712013000200005

Day, D. V., \& Dragoni, L. (2015). Leadership development: an outcome-oriented review based on time and levels of analyses. Annual Review of Organizational Psychology and Organizational Behavior, 2, 133 156. doi: 10.1146/annurev-orgpsych-032414-111328

Dias, M. A. M. J., \& Borges, R. S. G. (2015). Estilos de liderança e desempenho de equipes no setor público. Revista Eletrônica de Administração, 21(1), 200-221. doi: 10.1590/1413-2311.0542014.53468

Dionne, S. D., Gupta, A., Sotak, K. L., Shirreffs, K. A., Serban, A., Hao, C., ... Yammarino, F. J. (2014). A 25-year perspective on levels of analysis in leadership research. The Leadership Quarterly, 25(1), 6-35. doi: 10.1177/1059601111401017

Fonseca, A. M. O., Porto, J. B., \& Borges-Andrade, J. E. (2015). Liderança: um retrato da produção científica brasileira. Revista de Administração Contemporânea, 19(3), 290-310. doi:10.1590/1982-7849rac20151404.

Garg, A. K., \& Ramjee, D. (2013). The relationship between leadership styles and employee commitment at a parastatal company in South Africa. The International Business \& Economics Research Journal (Online), 12(11), 1411-1436. doi: 10.19030/iber.v12i11.8180 
Gnambs, T., \& Batinic, B. (2011). Convergent and discriminant validity of opinion leadership: multitrait-multimethod analysis across measurement occasion and informant type. Journal of Individual Differences, 32(2), 94-102. doi:10.1027/1614-0001/a000040.

Gottlieb, E. (2012). Mosaic leadership: charisma and bureaucracy in Exodus 18. Journal of Management Development, 31(9), 974-983. doi: 10.1108/02621711211259910

Hair Jr., J. F., Black, W.C., Babin, B. J., Anderson, R. E., \& Tatham, R. L. (2009). Análise multivariada de dados (6 $6^{\mathrm{a}}$ ed.). Porto Alegre: Bookman.

Hernandez, J. A. E., \& Voser, R. C. (2012). Validação da escala de liderança para o esporte: versão preferência dos atletas. Psicologia: Ciência e Profissão, 32(1), 142-157. doi: 10.1590/S141498932012000100011

Hersey, P., \& Blanchard, K. H. (1982). Psicologia para administradores: a teoria e as técnicas da liderança situacional (E. A. Royer, Trad.). São Paulo: Editora 10.1177/014920639702300306

Horn, J. L. (1965). A rationale and technique for estimating the number of factors in factor analysis. Psychometrika, 30(2), 179-185. doi: 10.1007/BF02289447

House, R. J., \& Delbecq, H. A. (1997). The social scientific study of leadership: quo vadis? Journal of Management, 23(3), 409-473. doi: 10.1177/014920639702300306

Hu, L., \& Bentler, P. (1999). Cut off criteria for fit indexes in covariance structure analysis: conventional criteria versus alternatives. Structural Equation Modelling, 6(1), 1-55. doi: 10.1080/10705519909540118

Hunt, J. B. (2010). Leadership style orientations of senior executives in Australia: senior executive leadership profiles: an analysis of 54 Australian top managers. Journal of American Academy of Business, 16, 207-218. Recuperado de http://hdl.handle.net/1959.13/1051376

Judge, T. A., \& Kammeyer-Mueller, J. D. (2012). Job Attitudes. Annual Review of Psychology, 63, 341-367. doi: 10.1146/annurev-psych-120710-100511

Larsson, G., \& Eid, J. (2012). An idea paper on leadership theory integration. Management Research Review, 35(3/4), 177-191. doi: 10.1108/01409171211210109.

Likert, R. (1961). New Patterns of Management. Nova lorque: McGraw-Hill.

Lins, M. A. P. B. E., \& Borges-Andrade, J. E. (2014). Expressão de competências de liderança e aprendizagem no trabalho. Estudos de Psicologia, 19, 159-168. doi: 10.1590/S1413-294X2014000300001

Liu, W., Lepak, D. P., Takeuchi, R., \& Sims Jr., H. P. (2003). Matching leadership styles with employment modes: strategic human resource management perspective. Human Resource Management Review, 13, 127-152. doi: 10.1016/S1053-4822(02)00102-X

Lok, P., \& Crawford J. (2004). The effect of organisational culture and leadership style on job satisfaction and organisational commitment: a cross-national comparation. The Journal of Management Development, 23(3), 321-338. doi: 10.1108/02621710410529785

Manning. T. (2013). A "contingent" view of leadership: 360 degree assessments of leadership behaviours in different contexts. Industrial and Commercial Training, 45(6), 343-351. doi: 10.1108/ICT-02-2013-0014

Melo, E. (2004). Escala de Avaliação do estilo gerencial (EAEG): desenvolvimento e validação. Revista Psicologia: Organizações e Trabalho, 4(2), 31-62. Recuperado de https://periodicos.ufsc.br/index.php/ rpot/article/view/7636

Miles, J., \& Shevlin, M. (2001). Applying regression \& correlation. A guide for students and researches. Londres: SAGE Publications.
Parry, K., Mumford, M. D., Bower, I., \& Watts, L. L. (2014). Qualitative and historiometric methods in leadership research: a review of the first 25 years of The Leadership Quarterly. The Leadership Quarterly, 25(1), 132-151. doi: 10.1016/j.leaqua.2013.11.006

Rodrigues, A. O., Ferreira, M. C., \& Mourão, L. (2014). O fenômeno da liderança: uma revisão das principais teorias. Fragmentos de Cultura, 23, 587-601. Recuperado de http://seer.ucg.br/index.php/ fragmentos/article/view/3556/2061

Romero, E., Villar, P., Gómez-Fraguela, J. A., \& López-Romero, L. (2012). Measuring personality traits with ultra-short scales: a study of the Ten Item Personality Inventory (TIPI) in a Spanish sample. Personality and Individual Differences, 53(3), 289-293. doi: 10.1016/j.paid.2012.03.035

Sant'anna, A.S., Campos, M. S., \& Lótfi, S. (2012). Liderança: o que pensam executivos brasileiros sobre o tema? Revista de Administração Mackenzie, 13(6), 48-76. doi: 10.1590/S1678-69712012000600004

Schweizer, K. (2011). Some thoughts concerning the recent shift from measures with many items to measures with few items. European Journal of Psychological Assessment, 27(2), 71-72. doi:10.1027/1015-5759/a000056

Scott, C. (2010). Perceptions of leadership under conditions of environmental uncertainty. Journal of American Academy of Business, 16(1), 30-35.

Silva, A. P. C., \& Ferreira, M. C. (2009). Escala de satisfação geral no trabalho. In Instituto Brasileiro de Avaliação Psicológica (Org.), IV Congresso Brasileiro de Avaliação Psicológica. Resumos (p. 246). Campinas: Autor

Silva, N. S. O., \& Mourão, L. (2015). A influência dos estilos de liderança sobre os resultados de treinamento. Estudos e Pesquisas em Psicologia, 15(1), 260-283. Recuperado de http://pepsic.bvsalud.org/scielo.php?script=sci_ arttext\&pid=S1808-42812015000100015\&lng=pt\&tlng=pt.

Silva, W., Laros, J., \& Mourão, L. (2007). Desenvolvimento e validação de escalas para avaliação da atuação gerencial. Revista Psicologia: Organizações e Trabalho, 7(1), 7-30. Recuperado de http:// pepsic.bvsalud.org/scielo.php?script=sci_arttext\&pid=S1984$-66572007000100002 \&$ Ing=pt\&tlng=pt.

Silva Junior, A., Oliveira, P. M. S., \& Vasconcelos, K. C. A. (2010). Desenvolvimento organizacional e a formação de lideranças: um estudo no setor de papel e celulose. Revista de Administração FACES Journal, 9, 15-31. doi: 10.21714/1984-6975FACES2010V9N2ART179

Souza, E. C. L., Lopez, G. S., Jr., Bornia, A. C., \& Alves, L. R. R. (2013). Atitude empreendedora: validação de um instrumento de medida com base no modelo de resposta gradual da teoria da resposta ao item. Revista de Administração Mackenzie, 14(5), 230-251. doi: 10.1590/S1678-69712013000500009

Spicker, P. (2012). "Leadership": a perniciously vague concept. International Journal of Public Sector Management, 25(1), 34-47. doi: 10.1108/09513551211200276

Sun, P.Y.T., \& Anderson, M. H. (2012). The combined influence of top and middle management leadership styles on absorptive capacity. Management Learning, 43, 25-51. doi: 10.1177/1350507611405116

Tabachinick, B. G., \& Fidell, L. S. (2007). Using multivariate statistics. (5 ed.). Nova lorque: Harper Collins.

Tupinambá, A. (2012). Empreendedorismo e liderança: perspectivas e desafios atuais. Revista Psicologia: Organizações e Trabalho, 12(1), 73-84. Recuperado de http://pepsic.bvsalud.org/scielo.php?script=sci_ arttext\&pid=S1984-66572012000100007\&lng=pt\&tlng=pt.

Tuuli, M. M., Rowlinson, S., Fellows, R., \& Liu, A. M. M. (2012). Empowering the project team: impact of leadership style and team context. Team Performance Management, 18(3/4), 149-175. doi: $10.1108 / 13527591211241006$ 
Velicer, W. F. (1976). Determining the number of components from the matrix of partial correlations. Psychometrika, 41(3), 321-327. doi: 10.1007/BF02293557

Wu, M. (2014). Verifying the influences of leadership styles upon organizational performances: balance-scored card implementation as a moderator. Journal of International Management Studies, 9(1), 25-37. Recuperado de http://www.jimsjournal.org/3\%20Mei-Fen\%20Wu.pdf
Yukl, G. (1989) Managerial leadership: a review of theory and research. Journal of Management, 15, 215-228. doi: $10.1177 / 014920638901500207$

Zanini, M.T.F., Santos, M. C. C., \& Lima, D. F.P.(2015). A influência do estilo de liderança consultivo nas relações de confiança e comprometimento no Batalhão de Operações Policiais Especiais do Rio de Janeiro. Revista de Administração, 50(1), 105-120. doi: 10.5700/rausp1187

Luciana Mourão, Doutora em Psicologia (PST), pela Universidade de Brasília (UnB), é Professora do Programa de Pós-Graduação em Psicologia da Universidade Salgado de Oliveira (UNIVERSO). Endereço para correspondência: Rua Marechal Deodoro, 217 - 2ํㅡㄴ Andar, Programa de Pós-Graduação em Psicologia, Universidade

Salgado de Oliveira - Campus Niterói, CEP: 24030-060, Fone/ Fax: (21) 2138-4926. E-mail: mourao.luciana@gmail.com

Cristiane Faiad, Doutora em Psicologia Social, do Trabalho e das Organizações (PSTO), pela Universidade de Brasília (UnB), é Professora Adjunta da Universidade de Brasília (UnB). E-mail: crisfaiad@gmail.com

Francisco Antonio Coelho Junior, Doutor em Psicologia Social, do Trabalho e das Organizações (PSTO), pela Universidade de Brasília (UnB), é Professor Adjunto do Departamento de Administração e Professor do Programa de Pós-Graduação em Administração (PPGA) da Universidade de Brasília (UnB). E-mail: fercoepsi@gmail.com

Recebido em 26.Jun.15 Revisado em 05.Ago.16 Aceito em 17.Out.16 